Documentation et bibliothèques

DOCUMENTATION BIBLIOTHEQUES

\title{
Mémoire à la Commission parlementaire de la Justice de l’Assemblée nationale du Québec
}

\section{Gaston Bernier}

Volume 21, numéro 2, juin 1975

URI : https://id.erudit.org/iderudit/1055498ar

DOI : https://doi.org/10.7202/1055498ar

Aller au sommaire du numéro

Éditeur(s)

Association pour l'avancement des sciences et des techniques de la documentation (ASTED)

ISSN

0315-2340 (imprimé)

2291-8949 (numérique)

Découvrir la revue

Citer cet article

Bernier, G. (1975). Mémoire à la Commission parlementaire de la Justice de l'Assemblée nationale du Québec. Documentation et bibliothèques, 21(2), 73-76. https://doi.org/10.7202/1055498ar
Résumé de l'article

Le mémoire présenté par le comité mixte ASTED/CBPQ/ABQ-QLA à la Commission parlementaire de la Justice de l'Assemblée nationale dans le cadre de la discussion du projet de loi sur les droits et les libertés de la personne comprend deux recommandations principales : d'abord que le droit à l'information soit incorporé aux libertés fondamentales comme garantie de l'exercice des autres libertés, puis que la documentation gouvernementale (documents administratifs, rapports d'enquête, arrêtés en conseil) soit considérée comme publique.
Tous droits réservés (C Association pour l'avancement des sciences et des techniques de la documentation (ASTED), 1975
Ce document est protégé par la loi sur le droit d'auteur. L'utilisation des services d'Érudit (y compris la reproduction) est assujettie à sa politique d'utilisation que vous pouvez consulter en ligne.

https://apropos.erudit.org/fr/usagers/politique-dutilisation/ 


\section{Mémoire à la Commission parlementaire de la Justice de l'Assemblée nationale du Québec}

Le mémoire présenté par le comité mixte ASTED/CBPQ/ABQ-QLA à la Commission parlementaire de la Justice de l'Assemblée nationale dans le cadre de la discussion du projet de loi sur les droits et les libertés de la personne comprend deux recommandations principales: d'abord que le droit à l'information soit incorporé aux libertés fondamentales comme garantie de l'exercice des autres libertés, puis que la documentation gouvernementale (documents administratifs, rapports d'enquête, arrêtés en conseil) soit considérée comme publique.

The written proposals submitted by the mixed committee ASTED/CBPQ/ $A B Q-Q L A$ to the parliamentary Commission on Justice of the National Assembly as far as the bill on the rights and freedom of the individual is concerned insist that the right to information be incorporated in the basic liberties as a guarantee of other liberties, and that the governmental documentation (administrative documents, enquiry reports, decrees in council) be open to the public.

El informe presentado por el comité mixto ASTED/CBPQ/ABQ-QLA a la comisión parlamentaria de justicia de la Asamblea nacional en el contexto de la discusión del proyecto de ley sobre los derechos y libertades del individuo comprende dos recomendaciones principales: primero, que el derecho a la información sea incluido en las libertades fundamentales como garantía del ejercicio de las otras libertades; segundo, que la documentación gubernamental (documentos administrativos, informes sobre investigaciónes, decretos) sea considerada como publica.

\section{Présentation}

Le 22 janvier dernier, l'ASTED, la CBPQ et I'ABQ/QLA présentaient, par l'intermédiaire du comité mixte sur la liberté intellectuelle, un mémoire à la Commission parlementaire de la Justice de l'Assemblée nationale au sujet du projet de loi numéro 50 dont le nom officiel est: "Loi sur les droits et libertés de la personne».

La présentation de ce mémoire découlait du mandat assigné au comité qui fut formé par les trois groupements en juin 1974: le comité devait, selon le deuxième article du mandat, "travailler en collaboration avec la Ligue des Droits de l'Homme afin de faire insérer dans l'éventuelle loi québécoise des droits de l'homme des articles concernant la liberté intellectuelle sous l'aspect particulier du droit à l'information».
Le projet de loi fut rendu public le 20 octobre 1974. Par, la suite, les membres du comité se réunirent à trois reprises entre le 6 novembre 1973 et le 15 janvier 1975 pour préparer le mémoire.

Dès le début, les membres du comité choisirent de préparer un court mémoire qui aborderait uniquement les problèmes concernant directement la profession. Ils savaient cependant qu'il aurait été possible d'élargir la portée du mémoire et de formuler des recommandations plus générales. D'ailleurs, l'ampleur de mémoires tels ceux présentés par la Ligue des Droits de l'Homme et le Réseau d'action et d'information pour les femmes prouvent bien cette possibilité. 
Le mémoire du comité comprend deux recommandations fort simples:

1) que le droit à l'information soit incorporé aux libertés fondamentales;

2) que la documentation gouvernementale soit considérée comme publique.

II n'est pas possible, présentement, de mesurer l'impact de notre mémoire, ni celui des mémoires des autres organismes. On le pourra au cours de la session qui a débuté en mars 1975 alors que les parlementaires auront à continuer l'étude du projet de loi. Cependant, il est presque certain que le ministre de la justice, parrain du projet, apportera des modifications relativement au droit à l'information même s'il est limité par le fait que ce texte législatif a déjà franchi l'étape de la seconde lecture. Par contre, on ne peut raisonnablement prévoir, pour le moment, une libéralisation relativement à l'accessibilité et à la disponibilité de la documentation gouvernementale en général. II faudra sans doute y revenir un jour.

\section{Gaston Bernier}

Bibliothèque de la Législature

Assemblée nationale

Québec

\section{Mémoire}

\section{Le comité mixte}

Le comité mixte ASTED/ABQ-QLA/ $C B P Q^{\prime}$ sur la liberté intellectuelle a été créé à l'été de 1974 par les trois associations de bibliothécaires œuvrant au Québec. Deux membres de chacune des trois associations, nommés par leur conseil de direction respectif, forment le comité.

Les associations de bibliothécaires ont confié au comité la mission d'étudier les cas d'atteinte à la liberté intellectuelle au Québec, de proposer des solutions et d'agir comme groupe de vigilance. Le comité doit également répandre parmi les membres des trois associations et même parmi tous les employés des bibliothèques le désir de défendre les libertés de l'esprit et leur en offrir la possibilité. À cet effet, le comité a déjà présenté aux conseils de direction des associations une charte des droits du lecteur. Les associations ont également assigné aux membres du comité la tâche d'étudier la charte québécoise des droits de l'homme, c'est-à-dire le texte connu aujourd'hui sous le nom de "Loi sur les droits et libertés de la personne" et de faire connaître le point de vue de l'ensemble de la profession sur ledit texte.

Le comité, avons-nous écrit, fut mis sur pied grâce à l'initiative de trois associations. Décrivons-les brièvement. L'ASTED

1. ASTED: Association pour l'avancement des sciences et des techniques de la documentation; ABQ-QLA: Association des bibliothécaires du Québec - Quebec Library Association; CBPQ: Corporation des bibliothécaires professionnels du Québec. ou Association pour l'avancement des sciences et des techniques de la documentation, est à la fois la plus ancienne et la plus jeune puisqu'elle a pris, en 1973, la relève de I'A.C.B.L.F. ou Association canadienne des bibliothécaires de langue française, qui existait auparavant. L'ASTED compte aujourd'hui plus de 1200 membres (bibliothécaires, bibliotechniciens, éditeurs, libraires, documentalistes, informaticiens) répartis sur tout le territoire québécois. La Corporation des bibliothécaires professionnels du Québec fut créée en 1968 par une loi de l'Assemblée nationale (Lois du Québec, 1969, ch. 105). Au-delà de 700 professionnels de la bibliothéconomie sont membres de cet organisme. Le troisième groupe, l'Association des bibliothécaires du Québec (Quebec Library Association) regroupe environ trois cents bibliothécaires surtout concentrés dans la région métropolitaine de Montréal.

\section{Recommandations}

Les recommandations des membres du comité touchent uniquement le domaine des libertés de l'esprit. Les droits judiciaires et politiques, les droits économiques et sociaux, le problème de la discrimination intéressent également les bibliothécaires. Nous espérons que les membres de l'Assemblée nationale, par l'intermédiaire de la Commission permanente de la Justice, auront la possibilité de discuter avec d'autres groupes spécialisés de ces problèmes et de ces droits qui sont loin de nous laisser indifférents. Mais notre domaine d'activité 
nous familiarise davantage avec les libertés intellectuelles.

A titre de bibliothécaires, nous nous sentons particulièrement concernés par toutes les questions relatives aux libertés intellectuelles. Les bibliothécaires ont, depuis toujours, la responsabilité de fournir des documents d'appui sur tous les points de vue, de mettre à la disposition des lecteurs les œuvres les plus diverses et les plus opposées. De plus, les bibliothécaires ne tiennent compte que des besoins du lecteur; sa religion, son origine ethnique et même ses opinions politiques leur importent peu. Agissant ainsi, les bibliothécaires croient servir la liberté intellectuelle.

Aussi, aujourd'hui, se croient-ils concernés par le projet de loi numéro 50 .

a) La première préoccupation des bibliothécaires du Québec est de voir ajouter, aux libertés fondamentales énumérées à l'article 3, le droit à l'information.

Les auteurs du projet de loi reconnaissent plusieurs libertés intellectuelles: la liberté de conscience, la liberté de religion, la liberté d'opinion, la liberté d'expression et la liberté de l'instruction. Mais, sans la liberté d'information, le respect de ces mêmes droits restera très hypothétique.

Pour deux raisons surtout. D'abord, parce que sans liberté d'information, il est loin d'être sûr que les violations des droits énumérés dans l'article 3 pourront être dénoncées. Ensuite, parce que la liberté d'information est la condition nécessaire à la prise de conscience de l'existence des autres libertés. La personne ne peut pas jouir d'une liberté qu'elle ignore comme elle ne pourra pas dénoncer l'injustice dont elle est la victime inconsciente.

Nous considérons que la liberté d'information est la première des libertés intellectuelles. Sans elle, la liberté d'opinion ne pourra pas fleurir, pas plus que la liberté de religion ou la liberté d'expression.

La liberté d'exprimer ses croyances profondes et ses idées par la parole, par l'écrit ou par tout autre mode de communication perd son sens si, dans le même temps, on nie à d'autres personnes le droit d'accéder à ces divers modes d'expression.

Nous avons été les témoins impuissants de quelques cas de censure dans les bibliothèques du Québec au cours des der- nières années. En octobre 1970, la Sûreté du Québec se rendait à la Bibliothèque nationale pour confisquer les œuvres de Pierre Vallières. Plus tard, le comité de la bibliothèque publique de Cowansville exigeait le retrait de certains périodiques de la collection de la bibliothèque. Récemment, la Church of Scientology menaça la direction de Sir George Williams University de poursuite judiciaire si la direction de la bibliothèque ne retirait pas de sa collection un livre très critique à son égard, etc.

Pour aider les bibliothécaires à lutter efficacement contre les censeurs et parce que la liberté d'information est une condition nécessaire au maintien des autres libertés intellectuelles, nous proposons aux membres de l'Assemblée nationale d'inclure, à l'article 3 , la «liberté d'information» et que l'article se lise comme suit:

"Tout être humain est titulaire des libertés fondamentales telles la liberté d'information, la liberté de conscience, la liberté de religion, la liberté d'opinion, la liberté d'expression, la liberté de réunion pacifique et la liberté d'association».

b) Les membres du comité mixte et ceux des associations représentées recommandent également aux législateurs québécois d'introduire, dans le projet de loi, un article dont le sens devrait être le suivant:

"Toute information recueillie, rassemblée et conservée par un organisme gouvernemental ou para-gouvernemental est disponible pour toute personne en faisant la demande, sauf dans les cas d'exception prévus."

Une telle proposition est assez nouvelle dans notre milieu. II ne faudrait pas en conclure qu'elle est sans objet et sans fondement.

En dépit du grand nombre de publications éditées et distribuées par les organismes gouvernementaux, les bibliothécaires doivent régulièrement faire face à des situations insolubles: les statistiques publiées sont trop globales et ne répondent pas toujours aux besoins des chercheurs qui veulent des statistiques pour un territoire restreint; parfois les publications d'un ministère ne constituent qu'une introduction générale pour l'étudiant qui prépare une thèse. 
Par contre, les dossiers conservés dans les ministères seraient d'une grande utilité, mais leur consultation est souvent impossible, la plupart du temps aléatoire.

Nous sommes conscients du fait que les dossiers gouvernementaux administratifs ne pourraient pas tous être consultés. Nous acceptons les justes restrictions et les exceptions. Nous souhaiterions que la Commission des droits de la personne soit habilitée à entendre les arguments des parties, à juger de leur bien-fondé et à rendre un jugement.

Cette solution s'inspire largement de la pratique américaine et de la loi du Public Information Act (Public Law 89487, approuvée le 4 juillet 1966). Cette loi reconnaît le droit à l'information gouvernementale et énumère neuf exceptions. Dans l'ensemble, toute personne peut obtenir un dossier ou un renseignement de l'administration fédérale à la condition de bien préciser sa question. Le refus du fonctionnaire de fournir l'information désirée peut être porté devant la cour fédérale et le poids de la preuve en revient au gouvernement.

L'introduction, chez nous, d'une telle mesure aurait également l'avantage de nous rapprocher de la sociale-démocratie suédoise. En Suède, "les théories mises de l'avant... soutiennent que le secret administratif, comme le souligne Me Jacques Prémont dans un article sur la publicité des documents officiels, va à l'encontre d'un véritable esprit démocratique et que, par conséquent, le public doit avoir accès en tout temps aux documents de l'autorité».2

En plus de favoriser l'accès aux dossiers de l'administration, notre recommandation implique également que le gouvernement ne retienne pas inutilement des rapports lui ayant été soumis et qui devraient normalement être rendus publics.
Les bibliothécaires regrettent, entre autre chose, que certaines tranches du rapport Dorion sur l'intégrité du territoire soient encore sous clé, que le rapport FANTUS, préparé à la demande du ministre de l'Industrie et du Commerce, n'ait pas été publié et que l'étude portant sur les conséquences pour l'économie québécoise de l'entrée de la Grande-Bretagne dans la CEE soit restée cachée. Nous pourrions multiplier les cas. Qu'on nous permette simplement de souligner, en plus, la disparition des études réalisées pour le compte de la Commission Rioux sur l'enseignement des arts, disparition dont la responsabilité reviendrait à un "certain membre du gouvernement du Québec».

De même, nous souhaitons, par cette résolution, que le gouvernement publie davantage d'arrêtés en conseil dans la Gazette officielle. Nous croyons que la proportion des règlements et arrêtés en conseil inédits reste trop importante. L'utilité de la Gazette officielle en est réduite alors qu'elle devrait tendre à l'exhaustivité. 